\title{
5 E-Health im Krankenhauswesen - Standortbestimmung und Ableitung strategischer Handlungsfelder durch Reifegradmodelle
}

\author{
Konrad Fenderich, David Matusiewicz, Vanessa Gieseler und \\ Steffen Fleßa
}

\section{Endlich: E-Health auch in Deutschland}

Im Dezember 2015 hat der Deutsche Bundestag das vom Bundesgesundheitsminister Hermann Gröhe eingebrachte und als „E-Health-Gesetz“ bekannte „Gesetz für sichere digitale Kommunikation und Anwendungen im Gesundheitswesen sowie zur Änderung weiterer Gesetze“ angenommen.

„Unter E-Health fasst man Anwendungen zusammen, die für die Behandlung und Betreuung von Patientinnen und Patienten die Möglichkeiten nutzen, die moderne Informationsund Kommunikationstechnologien (IKT) bieten“" Bundesministerium für Gesundheit 2015)

Dem Bundesministerium für Gesundheit zufolge beinhaltet das neue Gesetz „einen Fahrplan für die Einführung einer digitalen Infrastruktur mit höchsten Sicherheitsstandards und die Einführung nutzbringender Anwendungen auf der elektronischen Gesundheitskarte“ als „Voraussetzungen für die medizinische Versorgung der Zukunft" (Bundesministerium für Gesundheit 2017). So sollen beispielsweise mithilfe der Gesellschaft für Telematikanwendungen der Gesundheitskarte (gematik) und der Industrie bis Mitte 2018 „Arztpraxen und Krankenhäuser flächendeckend an die Telematik-Infrastruktur angeschlossen " und Patienten mit einer auf der elektronischen Gesundheitskarte gespeicherten elektronischen Akte „besser über ihre Diagnosen und Therapien informiert" (Bundesministerium für Gesundheit 2017) sein. 


\section{Das deutsche Krankenhauswesen ist rückständig}

Das Bundesministerium für Gesundheit unterstrich seinen Willen, den ausgegebenen Fahrplan von nun an mit voller Kraft zu verfolgen, indem es zehn Monate nach dem E-Health-Gesetz im Oktober 2016 eine von ihm beauftragte und großangelegte Studie zur Weiterentwicklung der E-Health Strategie vorstellte. Ein wichtiges Ergebnis dieser Studie war, dass die gesellschaftliche Akzeptanz für einen flächendeckenden Einsatz digitaler Anwendungen erhöht werden muss, da ansonsten ein „zögerliches Verhalten einzelner Akteure oder einzelner Akteursgruppen den Projekterfolg maßgeblich gefährden“"(Blachetta et al. 2016) kann.

Ungeachtet der hohen Aufmerksamkeit, die das Bundesministerium für Gesundheit dem Thema E-Health zukommen lässt, ist eben ein solch zögerliches Verhalten in Bezug auf die Digitalisierung besonders in der deutschen Krankenhauspolitik zu bemerken.

So greifen in anderen EU-Mitgliedsstaaten bereits seit knapp zehn Jahren gesetzliche Regelungen zur Implementierung von elektronischen Patientenakten. Im Jahr 2015 hatten der Weltgesundheitsorganisation zufolge zudem bereits 70\% der Länder Europas eine E-Health Gesetzgebung verabschiedet oder zumindest eine entsprechende Strategie verfolgt (Weltgesundheitsorganisation 2016).

\section{Fehlende Ressourcen im Krankenhauswesen als Innovationshemmnis}

Spiegelbildlich haben viele Krankenhäuser ihre Strategie (noch) nicht auf die Digitalisierung ausgerichtet und in Bezug auf die Umsetzung solcher Strategien werden in der Krankenhauspraxis Missstände deutlich. So verfügen lediglich rund ein Drittel der deutschen Krankenhäuser über eine Digitalstrategie, auch weil E-Health in der Krankenhausführung mehrheitlich noch nicht als Managementthema angesehen und dementsprechend anderen Themen untergeordnet wird. Verschiedene internationale Erhebungen stellen insgesamt fest, dass digitale Anwendungen in den deutschen Krankenhäusern unterdurchschnittlich wenig eingesetzt werden (Hübner 2014; Sabes-Figuera 2013). Damit nutzen die deutschen Krankenhäuser die vielfältigen strategischen Möglichkeiten der neuen Informations- und Kommunikationstechnologien nur unzureichend, obwohl diesen vorausgesagt wird in Zukunft zu einem kritischen Erfolgsfaktor für die Wettbewerbsfähigkeit der Krankenhäuser werden (Mühlbacher u. Pflügel 2009). Die Potenziale der Digitalisierung bleiben ungenutzt.

Da insbesondere fehlende Investitionsmittel als Hürde für eine weitere Digitalisierung im deutschen Krankenhauswesen angesehen werden, ist es umso dringender notwendig, die den Krankenhäusern zur Verfügung stehenden knappen Ressourcen auch in Bezug auf E-HealthProjekte möglichst effizient einzusetzen. 
5 E-Health im Krankenhauswesen - Standortbestimmung und Ableitung strategischer Handlungsfelder durch Reifegradmodelle

Um dies leisten zu können, sollte mit geeigneten Managementwerkzeugen zunächst der tatsächliche Stand der Digitalisierung in den deutschen umfassend und genau erhoben und analysiert werden. Anschließend können je nach Ergebnisausprägung richtungsweisende Investitionsempfehlungen zur Wahrung der aktuellen und zukünftigen Wettbewerbsfähigkeit der Häuser durch den Einsatz von E-Health abgeleitet werden.

\section{Standortbestimmung durch Reifegradmodelle}

In diesem Zusammenhang erscheinen insbesondere Reifegradmodelle hilfreich, welche richtig angewandt als Management- oder Assessmentmethode den Status Quo sowie die stufenweise Entwicklung von Mitarbeitern, der Kultur, der Prozesse, Strukturen, Objekte und der Technologien eines Krankenhauses ermitteln können. Ein Reifegradmodell wird dann als erfolgreich angesehen, wenn es das aktuelle Leistungsniveau eines Untersuchungsobjektes bewertet und gegenüber dem Leistungsniveau von Wettbewerbern benchmarkt, Entwicklungsstufen sowie Verbesserungsmaßnahmen ableitet und die Implementation und Umsetzung dieser Maßnahmen anleitet.

\section{Die Erhebung, Bewertung, Analyse und Einordnung des Einsatzes von digitalen Anwendungen in deutschen Krankenhäusern kann mithilfe von Reifegradmodellen durchgeführt werden.}

Typischerweise beinhaltet ein Reifegradmodell die vier Elemente Dimensionen, Beurteilungsinstrument, Reifeprinzip sowie Reifegrade. Die Dimensionen sind Fähigkeitsgebiete, welche das zu Untersuchungsfeld eingrenzen sowie thematisch zusammengehörende Aspekte untergliedern und zusammenzufassen. Eine Gestaltungsdimension besteht aus einem oder mehreren Gestaltungsobjekten, welche die grundlegenden Kriterien für die Bewertung der Reife innerhalb einer Gestaltungsdimension darstellen. Mithilfe eines qualitativen oder quantitativen Beurteilungsinstruments wird überprüft, ob und inwiefern die Gestaltungsobjekte eines Reifegrades erfüllt sind. Die Reifegrade wiederum beschreiben die Charakteristika und zu erfüllenden Gestaltungsobjekte der aufeinanderfolgenden Stufen der Reife des Untersuchungsobjektes. Das Reifeprinzip eines Modells definiert schlussendlich die Art und Weise, wie ein Reifegrad erreicht wird.

\section{Ausgeprägte Kritik an bestehenden Reifegradmodellen}

In der aktuellen wissenschaftlichen Literatur lassen sich bis zu vierzehn unterschiedliche Reifegradmodelle zur Bewertung der Informations- und Kommunikationstechnologien von Organisationen des Gesundheitswesens identifizieren. Der überwiegende Teil dieser Modelle fokussiert jedoch spezielle Einzelthemen wie die Reife der elektronischen Patientenakte, der IT-Infrastruktur oder der Interoperabilität von Systemen. Lediglich zwei Modelle ersuchen die Reife der gesamten Informations- und Kommunikationstechnologien im Krankenhaus zu erfassen: 
1. Das „Quintegra Maturity Model for electronic Healthcare“ unterscheidet sieben Reifegrade, in welchen zunehmend mehr Arbeitsgebiete und (nationale) Instanzen in den Digitalisierungsprozess eines Krankenhauses einbezogen werden. Jede Stufe stellt dabei höhere Anforderungen an die IT-Infrastruktur des Krankenhauses.

2. Das „IDC Healthcare IT (HIT) Maturity Model“ beschreibt die Reifeentwicklung eines Krankenhausinformationssystems anhand von fünf Reifegraden. Innerhalb der Reifegrade wird das Informationssystem eines Krankenhauses stufenweise mit mehr Fähigkeiten ausgestattet, bis das gesamte Krankenhaus in diesem System vernetzt ist.

Allerdings werden die in der Literatur bestehenden Reifegradmodelle teils heftig kritisiert, da diese nicht oder zumindest nur teilweise den Anforderungen an ein wissenschaftliches Reifegradmodell entsprechen. Insbesondere fehlen häufig eines oder mehrere der beschriebenen Merkmale eines fundierten Reifegradmodells. Auffällig ist, dass bei den publizierten Reifegradmodellen keine Angaben zum zugrundeliegenden Beurteilungsinstrument gemacht werden, was eine Anwendung der Reifegradmodelle in der Krankenhauspraxis unmöglich macht. Weiterhin werden auch der Entwicklungsprozess und die zugrundeliegende Methodik der Reifegradmodelle zumeist nicht beschrieben, wodurch eine Validierung dieser unmöglich wird. Zudem sind bei diesen Modellen oftmals die genaue Beschreibung und Gewichtung der Gestaltungsobjekte je Reifegrad sowie die Beschreibung eines systematischen Prozesses zur fundierten Erhöhung der Reife nicht hinterlegt (vgl. Carvalho et al. 2016).

"None of the identified models has a sufficiently broad scope covering all areas and subsystems of health care organizations. In this sense, a maturity model with a holistic approach including a comprehensive set of influencing factors is missing. In this perspective, a new model to fill the gap should be designed. This new model, should include the main influence factors with different weights depending on their relative importance and its development should be supported by rigorous scientific methods of conceptualization and validation". (Carvalho et al. 2016)

Aufgrund der ausgeprägten Kritik an den aktuell bestehenden Reifegradmodellen ist ein für den deutschen Krankenhaussektor allgemeingültiges Reifegradmodell, welches auf Basis von praktisch relevanten und wissenschaftlich fundierten Kriterien den Stand der Digitalisierung eines Krankenhauses erhebt und bewertet, erst zu entwickeln. Die Hauptziele, die mit der Entwicklung eines solchen Reifegradmodells verfolgt werden sollten, lauten zusammengefasst:

1. Erhebung eines differenzierten Stands der Digitalisierung im Sinne des Einsatzes von E-Health Anwendungen im Krankenhaus mithilfe eines geeigneten Beurteilungsinstrumentes,

2. Bewertung des Einsatzes von E-Health Anwendungen durch ein fundiertes, methodisches Scoring und Einordnung der Ergebnisse in vorab definierte Reifegrade und

3. Analyse und Benchmarking der Ergebnisse zur Ableitung von orientierenden Handlungsempfehlungen für Krankenhausgeschäftsführung, -träger und Politik. 
5 E-Health im Krankenhauswesen - Standortbestimmung und Ableitung

strategischer Handlungsfelder durch Reifegradmodelle

An die theoretische Konzeption eines neuen Reifegradmodells sollte auch immer eine anwendungsbezogene Evaluation anhand der gängigen Gütekriterien geknüpft sein, um Güte und Funktionalität des Modells bestätigen zu können, bevor es in der Praxis flächendeckend angewendet wird.

\section{E-Health ist mehr als die elektronische Patientenakte}

Ungeachtet der vielfältigen Anwendungsmöglichkeiten von E-Health im Krankenhaus wird häufig bereits die Einführung eines durchgängigen, papierlosen Dokumentationssystems als hinreichendes Kriterium angesehen, um ein Krankenhaus als vollständig digitalisiert bezeichnen zu können. Jedoch sei an dieser Stelle darauf hingewiesen, dass ein solches digitales Dokumentationssystem nur eine von vielen weiteren möglichen E-Health Anwendungen im Krankenhaus ist.

Diese Möglichkeiten zur Anwendung von E-Health können auf allen Ebenen eines Krankenhauses und entlang des gesamten Behandlungs- und Administrationspfades eines Patienten eingesetzt werden. In der Literatur werden wiederkehrend insbesondere die folgenden konkreten E-Health Anwendungen innerhalb von drei grob umrissenen Einsatzgebieten beschrieben:

1. Patientenverwaltung, beispielsweise durch Krankenhausinformationssysteme, elektronische Patienten- oder Gesundheitsakten und Patientenidentifikationssysteme,

2. Patientenversorgung, beispielsweise durch Telemedizin, klinische Entscheidungsunterstützungssysteme, Optimierung klinischer Behandlungspfade, Big und Smart Data (Informationsgewinnung aus Datenvernetzung), Präzisionsmedizin oder personalisierte Medizin, mHealth, Nutzung des Quantified Self („Selbstvermessung“ beziehungsweise Datenerhebung des Patienten), Prävention und Ambient Assisted Living (,umgebungsunterstütztes Leben") und

3. Prozessverbesserung, beispielsweise durch digitale Unterstützung der Managementfunktionen, eProcurement (Materialwirtschaft und Logistik), Qualitätsmanagement, Gesundheitskommunikation, Marketing und Social Media sowie eLearning.

Dementsprechend sollte auch ein entsprechendes Reifegradmodell die verschiedenen E-Health Anwendungsbereiche in den abzuprüfenden Dimensionen und Gestaltungsobjekten berücksichtigen. Die nachfolgend vorgeschlagene Einteilung der E-Health Anwendungsbereiche im Krankenhaus (s. Abb. 1) kann zudem die mittlerweile veraltete Einteilung der Cestaltungsbereiche innerhalb des 5-C's-Modells aus dem Jahr 2000 (vgl. Kacher et al. 2000) ersetzen.

\section{Zusammenfassung}

Internationale Erhebungen bescheinigen den deutschen Krankenhäusern im aktuellen Stand einen unterdurchschnittlichen Einsatz von E-Health Anwendungen. Gemessen an den tatsächlichen Möglichkeiten verbleiben erhebliche Verbesserungspotenziale. Im Zuge der Implementierung des neuen E-Health-Gesetzes und der ent- 


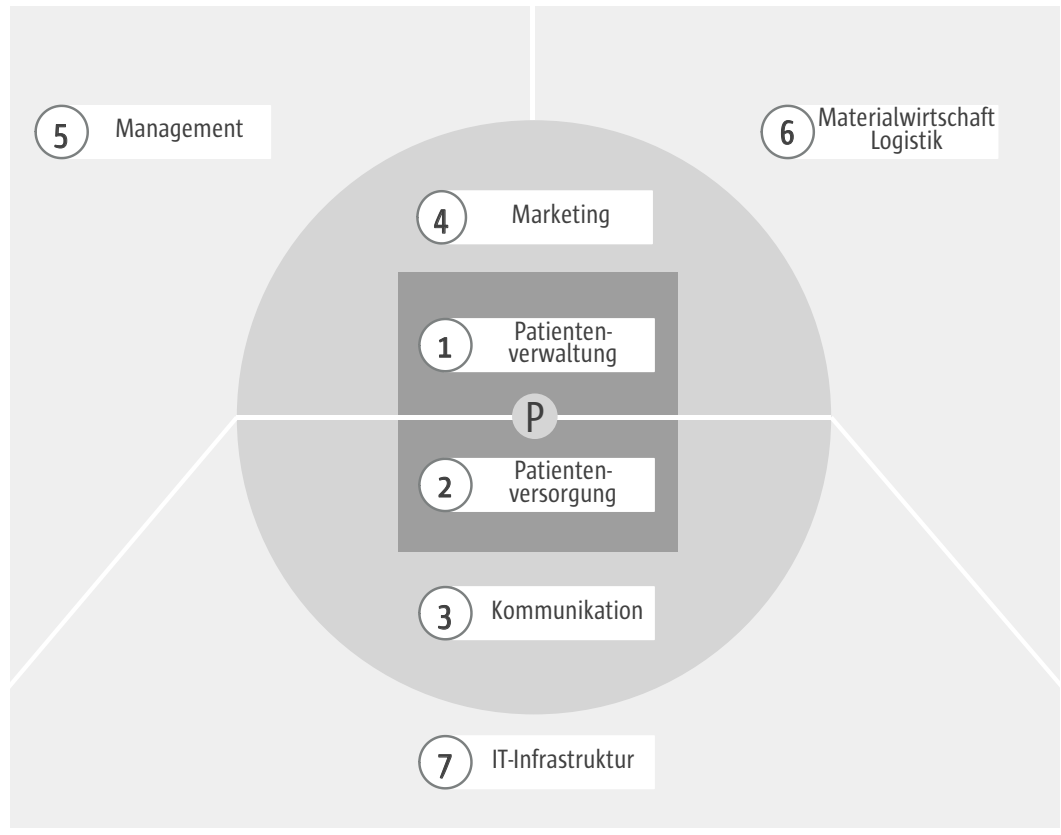

Anordnung der E-Health Anwendungsbereiche nach Interaktionshäufigkeit, Beeinflussungsstärke und räumlich-personeller Nähe zum Patienten:

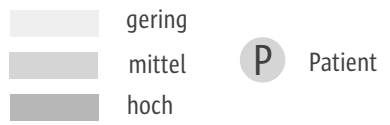

\section{Abb. 1 E-Health Anwendungsbereiche im Krankenhaus}

sprechenden Strategie des Bundesgesundheitsministeriums ist jedoch zu erwarten, dass sich der momentan geringe Stellenwert der Digitalisierung im Krankenhauswesen sowie begleitende Investitionssummen verändern werden. Diejenigen Bereiche, in denen eine Erhöhung des Einsatzes von E-Health Anwendungen im Krankenhaus betriebswirtschaftlich sinnvoll ist, sollten durch strategische Assessmenttools abgeleitet werden, um die knappen Ressourcen planvoll einzusetzen.

Durch die genaue Erhebung, Bewertung und Einordnung des aktuellen Digitalisierungsstands können Reifegradmodelle - zum Beispiel im Rahmen einer Selbst- oder Fremdbewertung - Investitionsentscheidungen zur zukünftigen Wahrung der Wettbewerbsfähigkeit durch zielgerichtete E-Health Anwendungen unterstützen.

\section{Literatur}

Blachetta F, Bauer M, Poerschke K, Bieber N, Solbach T, Leppert F, Greiner W, Bernnat R (2016) Weiterentwicklung der eHealth Strategie: Studie im Auftrag des Bundesministeriums für Gesundheit. Berlin: BMG

Bundesministerium für Gesundheit (2015) E-Health. Stand: 13. Januar 2015. URL: http://www.bundesgesundheitsministerium.de/service/begriffe-von-a-z/e/e-health.html (abgerufen am 01. Mai 2017)

Bundesministerium für Gesundheit (2017) Das E-Health-Gesetz. Stand: 15. März 2017. URL: http://www.bundesgesundheitsministerium.de/themen/krankenversicherung/e-health-gesetz/e-health.html (abgerufen am 01. Mai 2017) 
5 E-Health im Krankenhauswesen - Standortbestimmung und Ableitung strategischer Handlungsfelder durch Reifegradmodelle

Carvalho IV, Rocha A, Abreu A (2016) Maturity Models of Healthcare Information Systems and Technologies: a Literature Review. Journal of medical systems; 40(6): 131

Hübner U, Liebe I-D, Straede M-C, Thye I (2014) IT-Report Gesundheitswesen: Schwerpunkt IT-Unterstützung klinischer Prozesse. Befragung der bundesdeutschen Krankenhäuser. Hannover: Schriftenreihe des Niedersächsischen Ministeriums für Wirtschaft, Arbeit und Verkehr

Kacher C, Wiest A, Schumacher N (2000) E-Health: Chancen und Risiken für Ärzte, Patienten und Kostenträger. Zeitschrift für Allgemeinmedizin: 607-6013

Mühlbacher A, Pflügel R (2009) luK-Outsourcing im Krankenhaus: Das (digitale) Krankenhaus zwischen Integration und Fokussierung. In: Behrendt I, König H-J, Krystek U (Hrsg.). Zukunftsorientierter Wandel im Krankenhausmanagement. Berlin, Heidelberg: Springer-Verlag

Sabes-Figuera R, Abadie F, Maghiros I (2013) European Hospital Survey: Benchmarking deployment of e-health services (2012-2013) composite indicators on eHealth deployment and on availability and use of eHealth functionalities. Luxembourg: Publications Office EUR Scientific and technical research series

Weltgesundheitsorganisation (2016) From innovation to implementation: EHealth in the WHO European region. Copenhagen: WHO Regional Office for Europe 